DOI: $10.1002 /$ elan.201600556

Full Paper

N.^^T. Brannelly, A.^^J. Killard

$<?><?><?>$

\title{
A Printed and Microfabricated Sensor Device for the Sensitive Low Volume Measurement of Aqueous Ammonia
}

\author{
N. ${ }^{\wedge}$ T. Brannelly ${ }^{[\mathrm{a}]}$ and A.^^J. Killard*[a]
}

[a] <orgDiv/>Institute of Bio-Sensing Technology, Department of Applied Sciences, $<$ orgName/>University of the West of England, $<$ street $>$ Coldharbour Lane, $<$ city $/>$ Bristol, $<$ postCode/ $>$ BS16^1QY, $<$ country/ $>\mathrm{UK}$

E-mail: tony.killard@uwe.ac.uk

Ammonia
polyaniline
sensor
membrane
microfabrication
impedance

The measurement of low concentrations of ammonia in small sample volumes is required in biological, biomedical and environmental measurement applications. However, achieving this without instrumentation remains challenging. Here, sensor devices for the measurement of ammonia in a liquid were developed. These were based on fabrication of polyaniline nanoparticle films onto screen printed interdigitated electrodes using inkjet printing and their integration into a polymer microfabricated device with polytetrafluroethylene membrane and air flow path between the membrane and the sensor. Samples of ammonia in phosphate buffered saline of $52^{\wedge \wedge} \mu \mathrm{L}$ were measured using electrochemical impedance. While water vapour and ions from the buffer did result in a decrease in sensor impedance, this was eradicated by displacement of the headspace above the sensor with air. This, in combination with the adjustment of the sample to $\mathrm{pH}$ to 11 allowed the quantification of ammonia from 0 to 
$200^{\wedge} \wedge \mathrm{M}$ with a limit of detection of $25^{\wedge \wedge} \mu \mathrm{M}$. The device has the potential to be used for sensitive, low

volume measurement applications of ammonia at point-of-test and point-of-care.

\section{1_Introduction}

The application of electrochemical ammonia sensors is diverse and exists in many settings such as automotive [1--5], chemical [6--8], clinical [9--12], environmental [13--15] and food [16,^17] analysis. A number of ammonia sensors are available for these applications [18]. For example, ammonia is commonly used in refrigerant systems where it replaces environmentally unfriendly chlorofluorocarbon. It is being used in pharmaceuticals, food and beverage, air conditioning and in electric power plants. It is important to monitor ammonia in these settings as ammonia is toxic at high levels. Environmental concentrations of ammonia range from 0.1 to $200^{\wedge} \wedge \mathrm{ppm}$. Exposure to high concentrations of ammonia is associated with pulmonary related disorders. In the UK, the time-weighted Occupational Exposure Limit (OEL) for ammonia is $25^{\wedge} \mathrm{ppm}$ over an $8^{\wedge \wedge} \mathrm{hr}$ period and the Short Term Exposure Limit (STEL) is $35^{\wedge} \wedge$ ppm over a period of $15^{\wedge \wedge}$ min. However the lower limit of human perception of ammonia is $53^{\wedge} \wedge \mathrm{ppm}[19]$. Ammonia levels that are normal to humans are toxic to the aquatic environment. Pollution by agricultural run-off and natural decomposition of nitrogenous material in aquatic environments can be fatal to the aquatic populations as ammonia gas can penetrate biological barriers [20]. Ammonia is also monitored in biological matrices. It can be used to diagnose and monitor a range of pathological conditions such as liver dysfunction, abnormal nitrogen homeostasis, Helicobacter pylori infections [21]. Biological applications thus require measurement of ammonia at micromolar concentrations. Current blood ammonia quantification requires a laboratory blood test, sample treatment and analysis. In the UK, the most common technique for ammonia quantification is the glutamate dehydrogenase enzymatic reaction [21]. Few, if any of the techniques used are suitable for point of care (POC) testing. The development of a reliable and simple method for blood ammonia determination is essential for clinical diagnosis and management of patient progress in order to prevent further debilitating illnesses developing, and extending life.

Conducting polymers have gained significant scientific interest as an active layer in sensors due to their useful optical, electrical and magnetic properties [22]. Their utility has been further improved with new nanostructured fabrication and deposition techniques [23]. Polyaniline is a conducting polymer that is commonly applied to gas sensing due to its low cost and environmental stability. The principle feature of polyaniline as an ammonia sensor material lies in the emeraldine salt (ES) form's affinity with ammonia due to a distinct similarity of the coordinative roles of nitrogen atoms in polyaniline and ammonia. Nitrogen atoms of the polymer chains serve as adsorption centres for ammonia molecules. When the ES form of polyaniline $\left(\mathrm{PANIH}^{+}\right)$is interacting with ammonia the following reaction occurs: $<$ ffr $1>$

$<\mathrm{ffl}>\mathrm{PANIH}^{+}+\mathrm{NH}_{3} \square+\mathrm{NH}_{4}^{+}$

Ammonia deprotonates ES polyaniline to form energetically favourable ammonium ions [24]. It is this deprotonation that causes changes in the observable conductive behaviour. There are many polyaniline-based ammonia gas sensors in the literature, with a wide variety of fabrication techniques and sensing applications [11,^25--36]. Polyaniline can also be used for the detection of ammonia in aqueous environments. For example, an optical detector in combination with polyaniline-coated silica micro-capillaries was used for the detection of aqueous ammonia [37]. Optical sensing was also used to quantify aqueous ammonia with chemically synthesised polyaniline which was deposited onto glass substrates [38]. Another example is the amperometric determination of aqueous ammonia within a flow injection system which utilised electropolymerised polyaniline-modified platinum electrodes [39]. Our group has reported a number of chemical sensors and biosensors which have been produced using inkjet-printable polyaniline nanoparticle dispersions for the detection of ammonia in various matrices. 
The application of the inkjet polyaniline nanoparticles has been illustrated in a sensor for aqueous ammonia in refrigerant waste water via an amperometric flow injection system [40]. The system was found to have good performance and stability. An ammonia measurement probe for continuous monitoring of secondary refrigerants has also been developed. Impedimetric analysis was used to generate a strong calibration in the industrially relevant range of 0 to $100^{\wedge \wedge} \mathrm{ppm}$ [8]. All of these technologies are useful in an industrial stetting but are not suitable to miniaturised on-site field testing required for environmental analysis or POC bedside monitoring of pathological diseases related to ammonia because of the small sample volumes and low concentrations in complex matrices such as waste water, urine, blood etc.

In this work, we present the measurement of aqueous ammonia in low sample volumes $\left(52^{\wedge \wedge} \mu \mathrm{L}\right)$ at low concentrations ( 25 to $200^{\wedge \wedge} \mu \mathrm{M}$ ) using a printed polyaniline nanoparticle sensor integrated into a microfabricated microfluidic device. Measurement of ammonia gas from a liquid ammonia sample was performed using a membrane layer. The sensor was further made more sensitive and selective to ammonia by increasing the proportion of gaseous ammonia and removal of interferences from water vapour and ionic electrolytes.

\section{2_Experimental}

\section{1_Materials}

Ammonium persulphate (A7460), ammonium chloride (326372), aniline (132934; distilled and stored frozen under nitrogen before use), cellulose acetate dialysis tubing $22^{\wedge} \wedge \mathrm{mm}$ width (D9777) and $0.2^{\wedge \wedge} \mu \mathrm{m}$ polytetrafluoroethylene (PTFE) membrane filters (WHA10411411) were purchased from Sigma-Aldrich Company Ltd. (Dorset, UK). Potassium dihydrogen orthophosphate (P/4800/53), disodium hydrogen orthophosphate $(\mathrm{S} / 4520 / 53)$, sodium chloride $(\mathrm{S} / 3160 / 60)$, potassium chloride $(\mathrm{P} / 4280 / 53)$, sodium hydroxide $(\mathrm{S} / 4920 / 60)$ and sodium dodecyl sulphate $(\mathrm{S} / 5200 / 53)$ were purchased from Fisher Scientific UK Ltd (Loughborough, UK). Dodecylbenzene sulphonic acid (DBSA) soft type (D0989) was purchased from TCI Europe N.^^V. (Zwijndrecht, Belgium). Electrodag PF-410 silver conductive ink was purchased from NorCote International, Ltd. (Hampshire, UK). Sericol (ZT639) was purchased from Fujifilm. Preshrunk polyethylene terephthalate (PET) substrates of $175^{\wedge} \mu \mathrm{m}$ thickness were supplied by HiFi Industrial Film Ltd. (Hertfordshire, UK). Pressure sensitive adhesive (PSA) of $48^{\wedge} \mu \mathrm{m}$ thickness were supplied by Adhesives Research Inc. (Limerick, Ireland).

\subsection{Instrumentation}

Screen printing was performed with a semi-automated DEK-248 printing machine (DEK International, Dorset, UK). Ink-jet printing was carried out using a Dimatix Materials Printer DMP2831 with Dimatix Drop Manager DMP-2800 series software (Fujifilm Dimatix Inc., Santa Clara, CA). The MEMS-based Dimatix cartridge with $16^{\wedge \wedge}$ nozzles $\left(20^{\wedge \wedge} \mu \mathrm{m}\right.$ diameter) spaced at $254^{\wedge} \wedge \mathrm{m}$ was used. Centrifugation of polyaniline nanoparticles was carried out using a Beckman Coulter Allergra X22R Centrifuge with a Beckman C0650^^conical rotor head. A Graphtec CE5000--40 Craft Robo Pro cutting plotter (Graphtec GB Ltd., Wrexham, UK) and Robo Master-Pro software were used to prepare the PSA patterns for encapsulation of the sensor. All electrochemical protocols were performed on a Metrohm Autolab PGSTAT 128N potentiostat (Metrohm UK Ltd., Cheshire, UK) with Nova 1.6 software equipped with a FRA2 electrochemical impedance analyser. Unless otherwise stated all measurements were performed at room temperature $25 \pm 3^{\circ}$.

\subsection{Methods}




\subsection{1_Buffer}

$<?>0.1^{\wedge \wedge} \mathrm{M}$ phosphate buffered saline solution $(\mathrm{PBS})$ was prepared by dissolving $13.609^{\wedge \wedge} \mathrm{g}$ of potassium dihydrogen orthophosphate $\left(0.1^{\wedge \wedge} \mathrm{M}\right), 8^{\wedge \wedge} \mathrm{g}$ of sodium chloride $\left(0.137^{\wedge \wedge} \mathrm{M}\right)$ and $0.2^{\wedge \wedge} \mathrm{g}$ of potassium chloride $\left(2.7^{\wedge \wedge} \mathrm{mM}\right)$ in $1^{\wedge \wedge} \mathrm{L}$ deionised water, followed by the gradual addition of a $1^{\wedge \wedge} \mathrm{L}$ solution made up of $14.196^{\wedge \wedge} \mathrm{g}$ of disodium hydrogen orthophosphate $\left(0.1^{\wedge \wedge} \mathrm{M}\right), 8^{\wedge \wedge} \mathrm{g}$ of sodium chloride $\left(0.137^{\wedge \wedge} \mathrm{M}\right)$ and $0.2^{\wedge \wedge} \mathrm{g}$ of potassium chloride $\left(2.7^{\wedge \wedge} \mathrm{mM}\right)$ in deionised water, until $\mathrm{pH}^{\wedge \wedge} 7.4$ was reached.

\subsection{2_Electrode Fabrication via Screen Printing}

Silver screen printed interdigitated electrodes (IDEs) were fabricated via the method discussed in $[27, \wedge 40]$. This was carried out using a DEK-248 screen printer with a polyester screen with a mesh thickness $77^{\wedge \wedge} \mathrm{T}$ (filaments per $\mathrm{cm}$ ) and mounted at $45^{\circ}$ to the print stroke. Briefly, Electrodag PF-410 silver ink was deposited onto a $175^{\wedge} \wedge \mu \mathrm{m}$ thick PET substrate and cured at $120^{\circ}$ for $5^{\wedge \wedge} \mathrm{min}$. IDEs were designed in a two-electrode configuration with a working electrode and a common reference and auxiliary electrode. The electrodes were then modified with polyaniline nanoparticles.

\subsection{3_Polyaniline Nanoparticle Synthesis}

Polyaniline nanoparticles were synthesised according to the rapid mixing method [41]. Synthesis is described as follows: dodecylbenzene sulphonic acid (DBSA; $3.6^{\wedge \wedge} \mathrm{g}$ ) was made up to $40^{\wedge \wedge} \mathrm{mL}$ with deionised water, ammonium persulphate (APS; $0.36^{\wedge \wedge} \mathrm{g}$ ) dissolved in $20^{\wedge \wedge} \mathrm{mL}$ of the DBSA solution. The remaining DBSA solution was stirred at $20^{\circ}$ and aniline $\left(0.6^{\wedge \wedge} \mathrm{mL}\right)$ was added, followed quickly by the DBSA-APS solution. The mixture was left stirring for $2.5^{\wedge \wedge} \mathrm{hr}$. A $0.05^{\wedge \wedge} \mathrm{M}$ solution of sodium dodecyl sulphate (SDS) was prepared by dissolving $14.4^{\wedge \wedge} \mathrm{g}$ of SDS in deionised water. After stirring, $20^{\wedge \wedge} \mathrm{mL}$ of SDS was added to the dispersion which was centrifuged for $30^{\wedge} \mathrm{min}$ at $3000 \times \mathrm{g}$. The supernatant was finally dialyzed for $48^{\wedge} \mathrm{hr}$ against $500^{\wedge \wedge} \mathrm{mL}$ of SDS to remove excess material such as unwanted aniline. The final product was a dark green aqueous ink that was inkjet-printed onto the SPIDEs using a Dimatix Drop Manger Inkjet Printer.

\subsection{4_Inkjet Printing of Nanoparticles}

A Dimatix printer based on piezoelectric printed technology was used to print the pattern [27]. In brief, PANI nanoparticles were syringed into a Fujifilm Dimatix ink cartridge using a Thermo Syringe combined with an Acrodisc polyvinyl fluoride (PVDF) Syringe Filter $\left(0.45^{\wedge} \mu \mathrm{m}\right)$ and needle to inject $2^{\wedge \wedge} \mathrm{mL}$ of the polyaniline nanoparticulate solution. The Fujifilm Dimatix inkjet printer cartridges contain $16^{\wedge \wedge}$ nozzles that eject $10^{\wedge} \wedge \mathrm{pL}$ of ink. The cartridge was placed into the Dimatix inkjet printer at a head angle of $4.5^{\circ}$ where 10 layers were printed onto the silver IDEs. The operating conditions were optimised with a voltage of $22^{\wedge \wedge} \mathrm{V}$ and pitch density spacing set to $20^{\wedge \wedge} \mu \mathrm{m}$ and the substrate temperature was set to room temperature. After printing the sheets of dry sensors were lightly rinsed with deionised water to remove residual SDS, the sensors were then placed in a dry-heat oven at $70^{\circ}$ for $30^{\wedge \wedge} \min$.

\subsection{5_Ammonia Sensor Device Fabrication}

The printed ammonia sensors were further assembled into a device suitable for measuring ammonia in solution (Fig. ${ }^{\wedge} 1<$ figr $1>$ ). A silver screen-printed IDE deposited onto PET substrate was modified with ten layers of inkjet-printed polyaniline. A headspace of $247^{\wedge \wedge} \mathrm{mm}^{3}$ was created above the sensor using a $1.78^{\wedge \wedge} \mathrm{mm}$ thick 'o'-ring and gas-permeable PTFE membrane with a $0.2^{\wedge \wedge} \mu \mathrm{m}$ pore size and a 
diameter of $25^{\wedge \wedge} \mathrm{mm}$, bonded using layers of $48^{\wedge \wedge} \mu \mathrm{m}$ thick PSA. The distance between the sensor and membrane was $1.88^{\wedge \wedge} \mathrm{mm}$. A PSA lid layer was then attached to create a sample chamber with a capacity of $52^{\wedge \wedge} \mu \mathrm{L}$. Inlet and outlet ports were created using two hypodermic needles inserted into the o-ring at opposite sides of the device to allow the passage of compressed air over the sensor surface.

\subsection{6_Measurement of Ammonia}

Impedance measurements were carried out on the single use sensor devices. The sensors were typically exposed to ammonia as ammonium chloride in $0.1^{\wedge \wedge} \mathrm{M}$ PBS $\mathrm{pH}^{\wedge \wedge} 7.4$, unless otherwise stated. In order to change the $\mathrm{pH}$ of the solution to $\mathrm{pH}^{\wedge \wedge} 11.05^{\wedge \wedge} \mathrm{M} \mathrm{NaOH}$ was added to the sample at a ration of 1:25 and was left to incubate at room temperature for $10^{\wedge} \mathrm{min} .52^{\wedge} \wedge \mathrm{L}$ sample volume was left to incubate on the sensor surface for $15^{\wedge \wedge} \mathrm{min}$. Ammonia was measured at $10^{3 \wedge \wedge} \mathrm{Hz}, 5^{\wedge \wedge} \mathrm{mV}$ amplitude and a $1^{\wedge \wedge}$ s sampling rate for $161^{\wedge \wedge} \mathrm{s}$.

\section{3_Results and Discussion}

Whilst many methods have been published for the measurement of ammonia, few have shown the capability of performing measurements at concentrations and sample volumes compatible with biological processes [21]. Previous work using inkjet-printed films of polyaniline nanoparticles on IDEs in combination with electrochemical impedance has demonstrated the ability to detect ammonia down to several parts-per-billion in air and was almost as sensitive as instrumental methods such as mass spectrometry and laser spectroscopy [42]. It was believed that this was due to the excellent diffusional characteristics at the 2-dimensional, thin film sensor surface using impedance measurement. Such an approach might also be suitable for the measurement of ammonia in liquid samples. However, 2electrode impedimetric sensing is not suitable for liquid measurements which would short-circuit the system. Thus, to operate effectively, the liquid phase would require separation from the gas phase by an impermeable membrane layer that would allow diffusion of gas. PTFE has been shown to be suitable in such applications [8]. A simple, microfabricated sensor device was thus constructed to achieve this (Fig. $\wedge^{\wedge} 1<x f i g r l>$ ). However, it was not known how the device would behave in this new configuration, and detailed characterisation was performed.

\section{1_Characterisation Using Impedance Spectroscopy}

To assess the performance of the device for ammonia determination, the impedance spectroscopic characteristics of the device were initially assessed in air, PBS, and PBS containing $1^{\wedge \wedge} \mathrm{mM} \mathrm{NH}_{3}$. PBS is an aqueous biological buffer, containing sodium chloride and a phosphate buffering system that is widely used to mimic the behaviour of natural biological systems. Impedance spectroscopic characterisation took place across a frequency range of $10^{<\mathrm{M}->1}$ to $10^{5 \wedge \wedge} \mathrm{Hz}$ at amplitude of $5^{\wedge \wedge} \mathrm{mV}$. Figs. $\wedge^{\wedge} 2<$ xfigr $2>$ a to $c$ show the Nyquist, modulus and phase plots of the sensor in air, PBS and PBSammonia, respectively. The Nyquist plot and accompanying inset show that in all cases, the sensors behaved predominantly as a non-ideal resistor, with a small capacitive contribution. This can be more clearly seen in Fig. $\wedge^{\wedge} 2<$ figr $2>c$, with a decrease in phase angle occurring above approximately $10^{3 \wedge \wedge} \mathrm{Hz}$. However, below this frequency, all measurements showed predominantly resistive behaviour, which is fully in line with previously published data on these materials [42,^43].

What is most notable is that the impedance of the sensor in PBS was lower than that in air, suggesting that, while the PTFE membrane was impermeable to liquid water, water vapour with or without additional ions, was capable of traversing the membrane and reducing the impedance of the sensor to a level below its baseline in air [40,^42]. This is extremely problematic from an analytical perspective as it creates a non-linear region of measurement and does not allow for the use of an impedance measurement in air to be taken as a baseline. 
This issue is further illustrated in Fig. ${ }^{\wedge} 3<$ figr $3>$ which shows the impedance spectra of the devices in air and PBS containing ammonia at $0.1,0.5$ and $1^{\wedge \wedge} \mathrm{mM}$. Firstly, it is clear that the baseline resistance varies from device to device due to slight differences in layer film thickness and overall film resistance and thus, requires an impedance measurement in air to establish a baseline measurement prior to sample application. It can also be seen that while the presence of ammonia in PBS contributes to an increase in both real and, to a small degree, imaginary impedance, with increases in 656 and $13,668^{\wedge \wedge} \Omega$ for 0.5 and $1^{\wedge \wedge} \mathrm{mM}$, respectively at $10^{3 \wedge \wedge} \mathrm{Hz}$, at $0.1^{\wedge \wedge} \mathrm{mM}$, the decrease in impedance brought about by the PBS is greater in magnitude than the increase brought about by the presence of the ammonia, thus putting a limitation on its detectability.

Previous work has measured ammonia in refrigerant fluids in the gas phase using a sensor probe composed of a polyaniline sensor and a PTFE membrane with impedimetric measurement [8]. However, in that case, the concentrations of ammonia were much greater, being in the range of 5 to $100^{\wedge \wedge} \mathrm{ppm}\left(0.29\right.$ to $\left.5.87^{\wedge \wedge} \mathrm{mM}\right)$. At these concentrations, the impact of the matrix was not observed. The target detection limits in this work is to achieve micromolar levels of quantification which are at least an order of magnitude below this.

The source of the decrease in impedance from the sample matrix was further investigated. Sensor devices were exposed to deionised (DI) water, PBS, and PBS containing ammonia $\left(0.1,1\right.$ and $\left.5^{\wedge \wedge} \mathrm{mM}\right)$. Fig. ${ }^{\wedge} 4<$ figr $4>$ shows the ratios of the absolute impedances with respect to air $\left(Z / Z_{\text {air }}\right)$ at frequencies between $10^{<\mathrm{M}->1}$ and $10^{5 \wedge \wedge} \mathrm{Hz}$. It can be seen that DI water brought about a decrease in $|Z|$ across the frequency range. This resulted in a $Z / Z_{\text {air }}$ value of 0.98 which was approximately $320^{\wedge \wedge} \Omega$ below its baseline in air. However, PBS resulted in a larger $Z / Z_{\text {air }}$ decrease of 0.95 , corresponding to $1180^{\wedge \wedge} \Omega$ below its baseline. This additional decrease can only be due to ionic components of either the phosphate salts or sodium chloride being carried by the water vapour. It is widely known that small molecules such as helium, oxygen and carbon dioxide can permeate through PTFE membrane via gaps in the polymer structure. Other molecules of similar structure to fluorine have been known to permeate through by substitution of fluorine atoms on the polymer chain on the surface of the PTFE [44].

It is further illustrated in Fig. ${ }^{\wedge} 4<<$ figr $4>$ that at a concentration of $0.1^{\wedge \wedge} \mathrm{mM}$ ammonia, the impedance signal was at or below both the air and water baselines $(0.97)$ at $10^{3 \wedge \wedge} \mathrm{Hz}$. However, concentrations of 1 and $5^{\wedge \wedge} \mathrm{mM}$ in PBS exhibited ratiometric increases of 1.0 and 1.2 , respectively. Overall, changes in absolute impedance were greater at lower frequencies, particularly below $10^{3 \wedge \wedge} \mathrm{Hz}$. To reach the limits of detection desired, a means of eradicating matrix interference was required.

\subsection{Elimination of Matrix Effects}

Water vapour is a long-established interferent in many sensors, including previous polyaniline resistometric and impedimetric types and has often been a barrier to successful sensor development and implementation $[11, \wedge 40, \wedge 45]$. However, it is also well-established that water molecules interact with polyaniline in a different manner to ammonia. While there is a relatively strong association between polyaniline and ammonia, its interaction with water is relatively weak. In an aqueous environment, water molecules must always be in contact with the polymer. However, in air, they form an equilibrium between the gas and solid phases. Reducing the vapour pressure of water in the gas phase above the sensor would cause the water vapour on the sensor to diffuse into the gas phase in an attempt to reequilibrate the vapour pressure. Previous work using polyaniline sensors in breath analysis has shown that providing an air flow across the sensors after the application of a breath sample results in the effective displacement of the weakly-bound water molecules, while leaving the more tightly bound ammonia behind, thus allowing selective measurement of ammonia in a water vapour matrix [24].

In the current device, a means of displacing the gas in the headspace above the sensor was developed. This consisted of two hypodermic needles oppositely positioned as entry and exit points within the o-ring spacer (Fig. ${ }^{\wedge} 1<$ xfigr $1>$ ). This was connected to a compressed air line. The removal of the matrix signal using an air purge was investigated. An initial impedimetric baseline reading in air was taken across a frequency range of $10^{<\mathrm{M}->1}$ to $10^{5 \wedge \wedge} \mathrm{Hz}$. The sensor was then exposed to PBS for 
$15^{\wedge \wedge} \min$ before being removed. Subsequently, the sensor headspace was then purged with $5^{\wedge \wedge}$ psi air for $1^{\wedge \wedge}$ min and a final absolute impedimetric spectrum was then taken (Fig. ${ }^{\wedge} 5<$ figr $5>$ ). It was observed that at $1^{\wedge \wedge} \mathrm{kHz}$, a device with an initial baseline $|Z|$ of $528^{\wedge} \wedge \Omega$, upon exposure to PBS exhibited a decrease in $|Z|$ of $68^{\wedge \wedge} \Omega$ in impedance to $460^{\wedge \wedge} \Omega$, corresponding to a $Z / Z_{\text {air }}$ of 0.87 . Low frequencies $\left(<10^{3 \wedge \wedge} \mathrm{Hz}\right)$ typically correspond to the resistive properties of the device in which there is a significant decrease due to water vapour and electrolytes associated with PBS. The subsequent air purge recovered the initial baseline to $528^{\wedge} \wedge \Omega$ (corresponding to a $|Z|$ of 1.0). It was quite clear that the use of headspace displacement effectively eradicated any matrix effect. While it was predicted that this would be suitable for the removal of water molecules, it also appears to have removed any effects of ions, which may remain dissolved in the water vapour. In conclusion, the air purge successfully recovered the device upon exposure to PBS. Device recovery was considered a critical aspect in attaining the LOD. Returning the device to its initial baseline allowed increases in resistance associated low levels of ammonia to be observed without being concealed by matrix interference.

\section{3_Adjustment of Sample pH}

Ammonia has a $\mathrm{pKa}$ of 9 . When the $\mathrm{pH}$ of the solution is at neutral or physiological $\mathrm{pH}$ of approximately $7,99^{\wedge} \%$ of the ammonia in solution will exist as ammonium $\left(\mathrm{NH}_{4}^{+}\right)$. Reciprocally, at $\mathrm{pH}^{\wedge \wedge} 11,99^{\wedge} \%$ of ammonia in solution will exist as ammonia $\left(\mathrm{NH}_{3}\right)$ [46]. $\mathrm{NaOH}_{(\mathrm{aq})}$ is a strong base with a pKa of approximately 14. In this work, ammonia was typically prepared as ammonium chloride in $\mathrm{PBS}_{\mathrm{pH}}^{\wedge \wedge}$ 7.4. When sodium hydroxide is in solution with ammonium chloride it produces ammonia $\left(\mathrm{NH}_{3}\right)$ along with salt and water. $<\mathrm{ffr} 2>$

$<$ ff2 $>\mathrm{NH}_{4} \mathrm{Cl}+\mathrm{NaOH} \square \quad \mathrm{H}_{2} \mathrm{O}+\mathrm{NaCl}$

Numerous ammonia tests and kits employ a change in $\mathrm{pH}$ of the sample solution to increase the proportion of the measured $\mathrm{NH}_{3}$ [47]. Our system is also dependent on the mass of ammonia, as it is $\mathrm{NH}_{3}$ that diffuses as a gas from the sample to the sensor, and so increasing its proportionate concentration should increase the measurable change in impedance.

A volume ratio of $1^{\wedge}: \wedge 255^{\wedge \wedge} \mathrm{M} \mathrm{NaOH}$ to the sample was used to adjust the $\mathrm{pH}$ to 11 . This was performed by adding $2^{\wedge \wedge} \mu \mathrm{L}$ of $5^{\wedge \wedge} \mathrm{M} \mathrm{NaOH}$ to $50^{\wedge \wedge} \mu \mathrm{L}$ of the sample. The samples were then deposited onto sensors for a range of time intervals of 2 to $32^{\wedge \wedge} \mathrm{min}$ and their impedance responses compared to ammonia in $\mathrm{PBS}$ at $\mathrm{pH}^{\wedge \wedge} 7.4$ (Fig. 6). Firstly, it can be seen that increasing the $\mathrm{pH}$ of the sample to 11 did not cause an increase in impedance. This is critical, as polyaniline is also $\mathrm{pH}$ sensitive. While water vapour and accompanying ions appear to be able to traverse the membrane to the polyaniline, this did not appear to be possible for the $\mathrm{OH}^{-}$ions present from sodium hydroxide. Thus, any increase in signal would not be an artefact of a change in $\mathrm{pH}$. Secondly, in the presence of $1^{\wedge \wedge} \mathrm{mM}$ ammonia, adjustment of the $\mathrm{pH}$ did lead to a significant increase in impedance which increased for approximately $15^{\wedge \wedge} \mathrm{min}$. before reaching a plateau, and resulting in an approximately 5-fold increase. While the change in $\mathrm{pH}$ from 9 to 11 should theoretically lead to an approximately 100 -fold increase in the proportion of $\mathrm{NH}_{3}$ to $\mathrm{NH}_{4}{ }^{+}$, other processes such as rates of diffusion may limit this increase. However, the change in $\mathrm{pH}$ yielded a potentially 5 -fold improvement in the achievable limit of detection.

\section{4_Analytical Performance}

The fully optimised ammonia sensor devices were used for the quantitative measurement of ammonia at biologically relevant concentrations in a sample matrix of $\mathrm{PBS}, \mathrm{pH}^{\wedge} \wedge$ 7.4, and adjusted to $\mathrm{pH}^{\wedge \wedge} 11$. Ammonia was measured in the range of 25 to $200^{\wedge \wedge} \mu \mathrm{M}(n=3)\left(\right.$ Fig. ${ }^{\wedge \wedge} 7<$ figr $7>$ ). The relationship between ratiometric impedance $\left(Z / Z_{\text {air }}\right)$ and ammonia concentration produced excellent linearity across the range with an $\mathrm{R}^{2}$ of 0.9868 , a slope of 0.0043 , an intercept of 0.9562 and RSDs in the range of 0.8 to $4.2^{\wedge} \%$. The device achieved a LOD of $25^{\wedge \wedge} \mu \mathrm{M}$. 
Variability in sensor impedance was investigated (Fig. ${ }^{\wedge} 8<$ figr $8>$ ). Variations may be due to the variations in the inkjet printing process. For example, material in-homogeneity and/or nozzle blockages during deposition of the ten print layers may result in the observed variations. Inkjet-printed polyaniline ammonia sensors have been fabricated previously with 33\% RSD [42]. This value is 4.8 fold higher than that reported in this work $\left(6.9^{\wedge} \%\right)$ (Fig. ${ }^{\wedge} 8<$ xfigr $8>$ ). For comparison, commercial companies typically have a RSD of 3 to $5^{\wedge} \%$ [48]. To compensate for variation in baseline impedances of printed sensors, a ratiometric method has been employed by Hibbard et ${ }^{\wedge} a l$. (2013b). This method was established based on initial sensor baseline in air $\left(Z_{\text {air }}\right)$ and the response of the sensor to ammonia $(Z)$. Each individual sensor response was normalised $\left(Z / Z_{\text {air }}\right)$ with respect to its initial baseline $\left(Z_{\text {air }}\right)$ and its response to ammonia $(Z)$. It was found that the sensors maintained a constant sensitivity to ammonia when measured in this manner. This method was also adopted throughout this work to compensate for changes in initial sensor (baseline) impedance.

Table $^{\wedge \wedge} 1<$ tabr $1>$ presents a range of aqueous ammonia sensing systems. Previous work by Subramanian $e t^{\wedge}$ al. has demonstrated the measurement of aqueous ammonia down to $278^{\wedge \wedge} \mu \mathrm{M}$ [8]. However, their approach employed a continuous flow injection system (FIS), with large sample volumes and regeneration of the sensor in buffer between measurements. Khan $e t^{\wedge} \wedge a l$. produced sensors using UV-curable polyurethane acrylate 'organoclay nanohybrids'. These sensors determined aqueous ammonia across the analytical range of $0.05^{\wedge \wedge} \mu \mathrm{M}--0.05^{\wedge} \mathrm{M}$ and reached a very low LOD of near $0.0175^{\wedge} \wedge \mathrm{M}$. However, the transduction method used in this work was based on a time-consuming current-voltage technique which involved sweeping from $0--1.6^{\wedge \wedge} \mathrm{V}$. Discrimination between concentrations was observed at approx. $1^{\wedge \wedge} \mathrm{V}$ which is not an acceptable voltage for biological measurements

Analytical performance is obviously extremely important when developing sensors. However, a balance needs to be reached between performance and simple, low cost and easily mass produced sensors if commercialisation is to be realised. Many of the sensing systems listed in Table ${ }^{\wedge \wedge} 1<\mathrm{xtabr} 1>$ utilise complex bulky sensing systems such as FIS $[8, \wedge 39, \wedge 49, \wedge 50]$. The current technology offers a miniaturised, disposable, simple and easily mass produced device for low volume measurement for onsite aqueous ammonia testing. While the current device requires an air purge from an external source, such a function can be readily integrated into a device [51]. Further optimisation can also be achieved. Currently, two incubation steps are required for sample preparation ( $\mathrm{pH}$ change and device exposure 10 and $15^{\wedge} \wedge$ min, respectively) which may be further reduced and removed via the integration of alkaline reagent with the PTFE membrane. Further reductions in sample chamber size and volume are readily achievable.

\section{4_Conclusions}

The development and optimisation of a simple, low cost, printed disposable sensor device for the sensitive detection of ammonia in low volume liquid samples was demonstrated. An aqueous dispersion of polyaniline was deposited over a silver IDE using piezoelectric inkjet printing and integrated into a microfabricated, microfluidic assembly using a PTFE membrane layer. While use of the PTFE membrane allowed measurement of ammonia from liquid using an impedimetric, two-electrode interdigitated configuration, interference from water vapour and dissolved ions remained an issue. These interferents were eradicated using headspace displacement to allow selective measurement of ammonia. Adjustment of the $\mathrm{pH}$ of the sample from 7 to 9 also increased the impedance response fivefold. The resulting sensor device was capable of the measurement of ammonia in a $52^{\wedge \wedge} \mu \mathrm{L}$ sample in the range from 0 to $200^{\wedge \wedge} \mu \mathrm{M}$ with an LOD of $25^{\wedge \wedge} \mu \mathrm{M}\left(\mathrm{R}^{2}\right.$ of 0.9868 , a slope of 0.0043 , an intercept of 0.9562 and RSDs in the range of 0.8 to $4.2^{\wedge} \%$ ), making it widely suitable for environmental, biological, biomedical and point-of-care diagnostic applications.

$<$ litl $><$ jnl $>$ N. Docquier, S. Candel, Prog. Energy Combust Sci. 2002, 28, 107--150</jnl>. 
$<$ lit2 $><$ jnl $>$ T. ${ }^{\wedge}$ D. Durbin, R. ${ }^{\wedge}$ D. Wilson, J. ${ }^{\wedge}$ M. Norbeck, J. ${ }^{\wedge}$ W. Miller, T. Huai, S. ${ }^{\wedge} H$. Rhee, Atmos. Environ. 2002, 36, 1475--1482</jnl $>$.

$<$ lit3 $><$ jnl $>$ R. Moos, R. Muller, C. Plog, A. Knezevic, H. Leye, E. Irion, T. Braun, K.^^J. Marquardt, K. Binder, Sensor Actuat. B-Chem. 2002, 83, 181--189</jnl $>$.

$<$ lit4 $><$ jnl $>$ J. Riegel, H. Neumann, H.^^M. Wiedenmann, Solid State Ionics. 2002, 152, 783--800</jnl $>$. $<$ lit5 $><$ jnl $>$ S. Chaisitsak, Sensors. 2011, 11, 7127--7140</jnl $>$.

$<$ lit6><jnl $>$ R..$^{\wedge}$ E. delaHoz, D. ${ }^{\wedge}$ P. Schlueter, W.^^N. Rom, Am. J. Ind. Med. 1996, 29, 209--214</jnl $>$. $<$ lit7 $><$ jnl $>$ I. Kohl, J. Beauchamp, F. Cakar-Beck, J. Herbig, J. Dunkl, O. Tietje, M. Tiefenthaler, C. Boesmueller, A. Wisthaler, M. Breitenlechner, S. Langebner, A. Zabernigg, F. Reinstaller, K. Winkler, R. Gutmann, A. Hansel, J. of Breath Res. 2013, 7, 017110</jnl >.

$<$ lit8 $><$ jnl $>$ R. Subramanian, K. Crowley, A. Morrin, A. ${ }^{\wedge}$ J. Killard, Anal. Methods. 2013, 5, 134-$140</$ jnl $>$.

$<$ lit9 $><$ jnl $>$ W. Ament, J. ${ }^{\wedge}$ R. Huizenga, E. Kort, T. ${ }^{\wedge}$ W. van ${ }^{\wedge \wedge}$ der $^{\wedge \wedge}$ Mark, R. ${ }^{\wedge}$ G. Grevink, G. ${ }^{\wedge} J$. Verkerke, Int. J. Sports Med. 1999, 20, 71--77</jnl >.

$<$ lit10 $><$ jnl $>$ D. ${ }^{\wedge}$ J. Kearney, T. Hubbard, D. Putnam, Dig. Dis. Sci. 2002, 47, 2523--2530</jnl>. $<$ lit11 ><jnl>T. Hibbard, K. Crowley, F. Kelly, F. Ward, J. Holian, A. Watson, A.^^J. Killard, Anal. Chem. 2013, 85, 12158--12165</jnl $>$.

$<$ lit12><jnl $>$ O. ${ }^{\wedge}$ B. Ayyub, A. ${ }^{\wedge}$ M. Behrens, B. ${ }^{\wedge}$ T. Heligman, M. ${ }^{\wedge}$ E. Natoli, J.^^J. Ayoub, G.

Cunningham, M. Summar, P. Kofinas, Mol. Genet. Metab. 2015, 115, 95--100</jnl>.

$<$ lit13 $><$ jnl $>$ J.^^ W. Erisman, R. Otjes, A. Hensen, P. Jongejan, P. van ${ }^{\wedge}$ den $^{\wedge}{ }^{\wedge}$ Bulk, A. Khlystov, H. Mols, S. Slanina, Atmos. Environ. 2001, 35, 1913--1922</jnl $>$.

$<$ lit14><jnl>X. Zhu, M. ${ }^{\wedge \wedge}$ A. Nanny, E. ${ }^{\wedge}$ C. Butler, Water Res. 2008, 42, 2736--2744</jnl>. $<$ lit15 $><$ jnl $>$ M. Aleixandre, M. Gerboles, Nose 2012: 3rd International Conference on Environmental Odour Monitoring and Control. 2012, 30, 169--174</jnl>. 
$<$ lit16><jnl >G.^^${ }^{\wedge}$ H. Mount, B. Rumburg, J. Havig, B. Lamb, H. Westberg, D. Yonge, K. Johnson, R. Kincaid, Atmos. Environ. 2002, 36, 1799--1810</jnl $>$.

$<$ lit17><jnl $>$ S. Ampuero, J.^^${ }^{\wedge}$. Bosset, Sensor Actuat. B-Chem. 2003, 94, 1--12</jnl $>$.

$<$ lit18 $><$ jnl $>$ B. Timmer, W. Olthuis, A. van^^den^^${ }^{\wedge}$ Berg, Sensor Actuat. B-Chem. 2005, 107, 666-$667</$ jnl $>$.

$<$ lit19 $><$ jnl $>$ T. Bruning, R. Bartsch, H.^^M. Bolt, H. Desel, H. Drexler, U. Gundert-Remy, A. Hartwig, R. Jaeckh, E. Leibold, D. Pallapies, A. ${ }^{\wedge}$ W. Rettenmeier, G. Schlueter, G. Stropp, K. Sucker, G. Triebig, G. Westphal, C. van^^${ }^{\wedge}$ Thriel, Arch. Toxicol. 2014, 88, 1855--1879</jnl>.

$<$ lit20 $><$ jnl $>$ M. ${ }^{\wedge}$ A. Sutton, J.^^${ }^{\wedge}$ W. Erisman, F. Dentener, D. Moeller, Environ. Pollut. 2008, 156, 583$-604</$ jnl $>$.

$<$ lit21 $><$ jnl $>$ R. Barsotti, J. Pediatr. 2001, 138, S11-S19</jnl $>$.

$<$ lit22 $><$ book $>$ D. ${ }^{\wedge}$ C. Trivedi, Handbook of Organic Conductive Molecules and Polymers Volume 2 ,

Conductive Polymers: Synthesis and Electrical Properties, (Ed. H.^^S. Nalwa), John Wiley \& Sons, Inc., United Kingdom 1997, pp. $\wedge^{\wedge} 505--572</$ book $>$.

$<$ lit23 $><$ jnl $>$ B. Weng, R. ${ }^{\wedge}$ L. Shepherd, K. Crowley, A. ${ }^{\wedge}$ J. Killard, G. ${ }^{\wedge}$ G. Wallace, Analyst. 2010, $135,2779--2789</$ jnl $>$.

$<$ lit24><jnl $>$ A. ${ }^{\wedge}$ L. Kukla, Y.^^M. Shirshov, S.^^A. Piletsky, Sensor Actuat. B-Chem. 1996, 37, 135-$140</$ jnl $>$.

$<$ lit25><jnl $>$ Z. Jin, Y.^^X. Su, Y.^^X. Duan, Sensor Actuat. B-Chem. 2001, 72, 75--79</jnl>.

$<$ lit26><jnl $>$ D. ${ }^{\wedge}$ S. Sutar, N. Padma, D. ${ }^{\wedge}$ K. Aswal, S.^^K. Deshpande, S.^^${ }^{\wedge}$ K. Gupta, J.^^V. Yakhmi, Sensor Actuat. B-Chem. 2007, 128, 286--292</jnl $>$.

$<$ lit27><jnl $>$ K. Crowley, A. Morrin, A. Hernandez, E. O'Malley, P.^^${ }^{\wedge}$ G. Whitten, G. ${ }^{\wedge}$ G. Wallace, M.^^R. Smyth, A.^^J. Killard, Talanta. 2008, 77, 710--717</jnl>.

$<$ lit28><jnl $>$ M. Liu, C. Dai, C. Chan, C. Wu, Sensors. 2009, 9, 869--880</jnl $>$. $<$ lit29><jnl>G. Rizzo, A. Arena, N. Donato, M. Latino, G. Saitta, A. Bonavita, G. Neri, Thin Solid Films. 2010, 518, 7133--7137</jnl $>$. 
$<$ lit30 $><$ jnl $>$ M. Matsuguchi, T. Asahi, Sensor Actuat. B-Chem. 2011, 160, 999--1004</jnl>.

$<$ lit31><jnl>P. Stamenov, R. Madathil, J.^^M.^^D. Coey, Sensor Actuat. B-Chem. 2012, 161, 989-$999</$ jnl $>$.

$<$ lit32 $><$ jnl $>$ J.^^I. Dobroczynska, K. Grabas, Mater. Sci.-Poland. 2013, 31, 95--100</jnl $>$.

$<$ lit33 $><$ jnl $>$ S. ${ }^{\wedge}$ P. Basak, B. Kanjilal, P. Sarkar, A.^^P.^^${ }^{\wedge}$ F. Turner, Synth. Met. 2013, 175, 127-$133</$ jnl $>$.

$<$ lit34><jnl $>$ Y. Zhang, J.^^J. Kim, D. Chen, H. ${ }^{\wedge}$ L. Tuller, G. ${ }^{\wedge}$ C. Rutledge, Adv. Func. Mater. 2014, 24, 4005--4014</jnl $>$.

$<$ lit35><jnl $>$ D..$^{\wedge}$ K. Bandgar, S. ${ }^{\wedge}$ T. Navale, S. ${ }^{\wedge}$ R. Nalage, R. ${ }^{\wedge}$ S. Mane, F.^^J. Stadler, D. ${ }^{\wedge}$ K. Aswal, S.^^K. Gupta, V.^^B. Patil, J. Mater. Chem. C. 2015, 3, 9461--9468</jnl>.

$<$ lit36 $><$ jnl $>$ J. Kumar, M. Shahabuddin, A. Singh, S.^^P. Singh, P. Saini, S.^^K. Dhawan, V. Gupta, Sci. Adv. Mater. 2015, 7, 518--525</jnl >.

$<$ lit37><jnl>L. Florea, D. Diamond, F. Benito-Lopez, Anal. Chim. Acta. 2013, 759, 1--7</jnl $>$.

$<$ lit38 $><$ jnl $>$ J. Castrellon-Uribe, M.^^${ }^{\wedge}$ E. Nicho, G. Reyes-Merino, Sensor Actuat. B-Chem. 2009, 141, $40--44</$ jnl $>$.

$<$ lit39><jnl $>$ M. Trojanowicz, T.^^K.^^V. Krawczyk, M. Zmorzynska, L. Campanella, Electroanalysis. 1997, 9, 1062--1066</jnl $>$.

$<$ lit40 $><$ jnl $>$ K. Crowley, E. O'Malley, A. Morrin, M.^^R. Smyth, A. ${ }^{\wedge}$ J. Killard, Analyst. 2008, 133, $391--399</$ jnl $>$.

$<$ lit41 $><$ jnl $>$ O. Ngamna, A. Morrin, A.^^J. Killard, S. ${ }^{\wedge}$ E. Moulton, M. ${ }^{\wedge}$ R. Smyth, G. ${ }^{\wedge}$ G. Wallace, Langmuir. 2007, 23, 8569--8574</jnl $>$.

$<$ lit42 $><$ jnl $>$ T. Hibbard, K. Crowley, A. ${ }^{\wedge}$ J. Killard, Anal. Chim. Acta. 2013, 779, 56--63</jnl $>$. $<$ lit43><jnl>M. Kalaji, L.^^M. Peter, J. Chem. Faraday T. 1991, 87, 853--860</jnl>. $<$ lit44 $><$ other $>$ Corrosion Resistant Products Ltd., 2016 $<$ /other $>$. $<$ lit45 ><jnl $>$ S. Wu, F. Zeng, F. Li, Y. Zhu, Eur. Polym. J. 2000, 36, 679--683</jnl $>$. 
$<$ lit46 $><$ book $>$ P. Atkins, J. de ${ }^{\wedge \wedge}$ Paula, Physical Chemistry, Oxford University Press, UK 2009 $<$ /book $>$.

$<$ lit47><pat $>$ L. ${ }^{\wedge \wedge}$ J. Dobler, J.^^M. Gibbons, V.^^${ }^{\wedge}$ Y. Evtodienko, 2006, US Patent 7033839</pat $>$.

$<$ lit48 $><$ other $>$ DropSens, 2015, Innovative Technology for Miniturised Electrochemistry. Available from: http://www.dropsens.com/en/home.html</other $>$.

$<$ lit49 $><$ jnl $>$ L. Canale ${ }^{\wedge}$ Analegutierrez, A. Maquieira, R. Purchade, Analyst. 1990, 115, 1243-$1246</$ jnl $>$.

$<$ lit50><jnl $>$ X. ${ }^{\wedge}$ L. Su, B. ${ }^{\wedge} \mathrm{S}$. Yu, H. ${ }^{\wedge}$ W. Tan, X. ${ }^{\wedge}$ R. Yang, L. ${ }^{\wedge} H$. Nie, S. ${ }^{\wedge}$ Z. Yao, J. Pharm. Biomed. Anal. 1998, 16, 759--769</jnl $>$.

$<$ lit51 ><jnl>B. Jose, P. McCluskey, N. Gilmartin, M. Somers, D. Kenny, A.^^J. Ricco, N.^^J. Kent, L. Basabe-Desmonts, Langmuir. 2016, 32, 2820--2828</jnl $>$.

$<$ lit52><jnl $>$ S.^^${ }^{\wedge}$ B. Khan, M. ${ }^{\wedge}$ M. Rahman, E. ${ }^{\wedge}$ S. Jang, K. Akhtar, H. Han, Talanta. 2011, 84, 1005-$1010</$ jnl $>$.

$<$ lit53 $><$ jnl $>$ T. Abel, B. Ungerboeck, I. Klimant, T. Mayr, Chem. Cent. J. 2012, 6, 124</jnl $>$.

$<$ lit54 $><$ jnl $>$ T.^^L. Ling, M. Ahmad, L. ${ }^{\wedge}$ Y. Heng, Sensor Actuat. B-Chem. 2012, 173, 614--619</jnl $>$.

$<$ lit55><jnl $>$ L. ${ }^{\wedge} Y$. Heng, S. Alva, M. Ahmad, Sensor Actuat. B-Chem.. 2004, 98, 160--165</jnl $>$.

$<$ lit56><jnl>J. Laranjeira, W. deAzevedo, M. deAraujo, Anal. Lett. 1997, 30, 2189--2209</jnl>.

Received: August 10, 2016

Accepted: October 22, 2016

Published online: $<?><?><?>$

Table $^{\wedge \wedge} 1 \quad$ Analytical performance of a range of aqueous ammonia sensing systems. $<\mathrm{W}=3>$

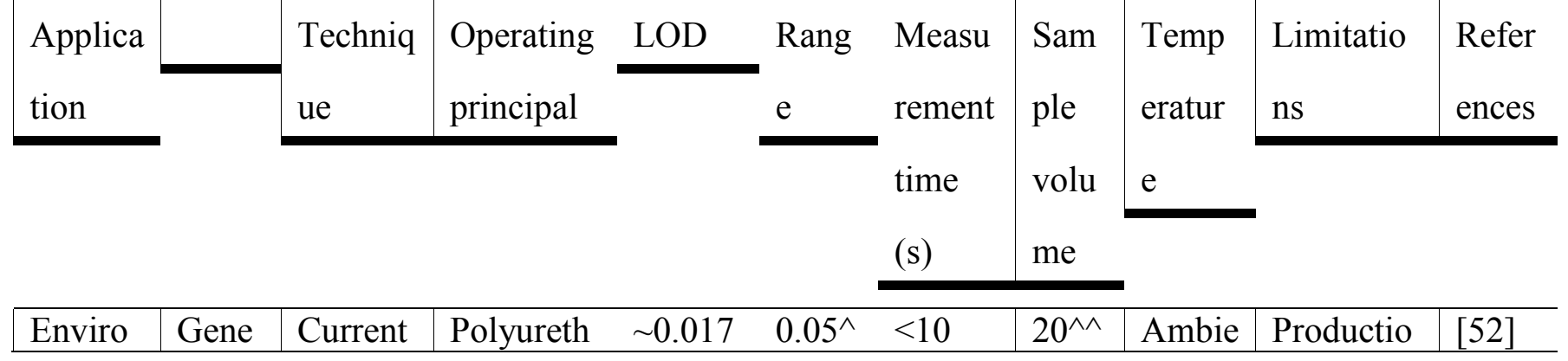




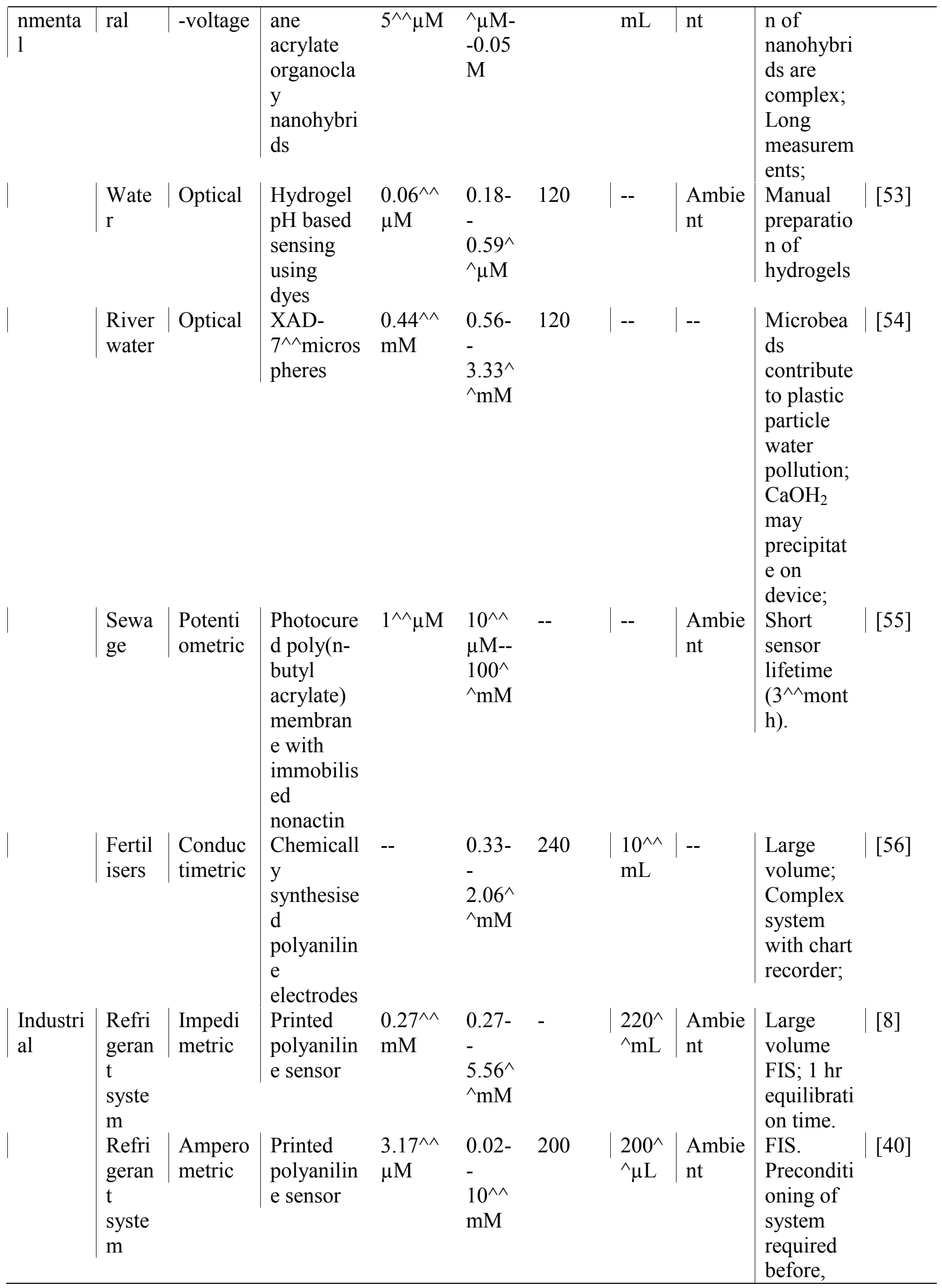




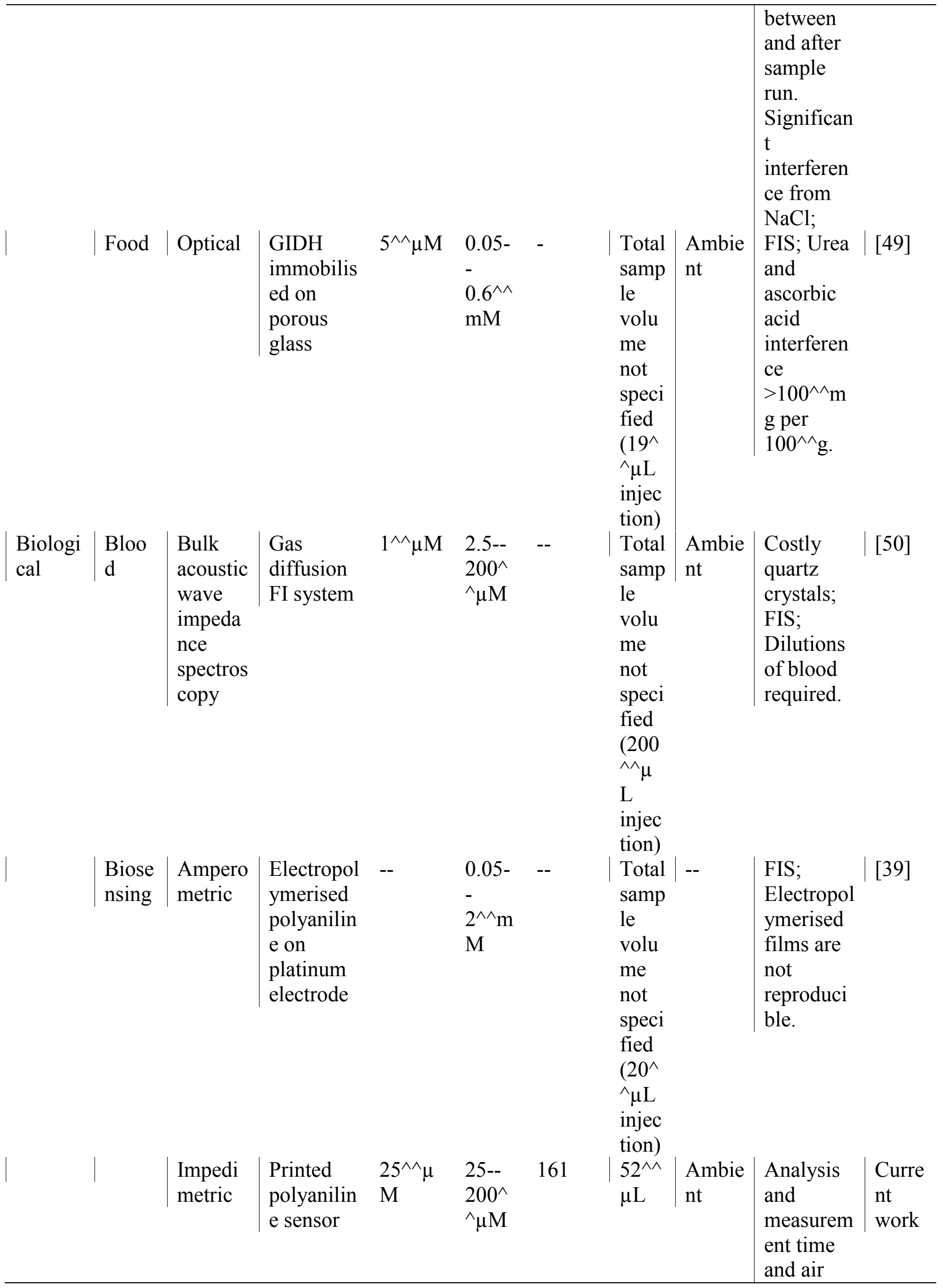


Figure $^{\wedge \wedge} 1 \quad$ (a) Design layout of the sensor device assembly. A silver screen-printed interdigitated electrode deposited onto PET substrate was modified with ten layers of inkjet-printed polyaniline. A headspace of $247^{\wedge \wedge} \mathrm{mm}^{3}$ was created above the sensor using a $1.78^{\wedge \wedge} \mathrm{mm}$ thick 'o'-ring and PTFE membrane, bonded using layers of $48^{\wedge} \wedge \mathrm{m}$ thick PSA. The sensor and headspace along with the PTFE membrane were encapsulated using PSA and a lid was attached to create a sample chamber with a capacity of $52^{\wedge} \wedge$ L. (b) Photograph of the sensor device. Left: Top view showing sample chamber and membrane. Right: Bottom view showing ammonia sensor. Also shown are two needles inserted into the $247^{\wedge \wedge} \mathrm{mm}^{3}$ headspace via the o-ring.

Figure $^{\wedge} 2$ Impedance spectra of the device in air $(\bullet)$, PBS $(\nabla)$ and $1^{\wedge} \mathrm{mM}$ ammonia in PBS $(\circ)$. (a) Nyquist, (b) Bode modulus, and (c) phase plots across a frequency range of $10^{<\mathrm{M}->1}$ to $10^{5 \wedge \wedge} \mathrm{Hz}$.

Figure $^{\wedge} 3$ Impedance spectroscopic analysis of the preliminary devices firstly in air $(\bullet)$ and secondly in DI water, PBS and 0.1, 0.5, $1^{\wedge \wedge} \mathrm{mM}$ ammonia in PBS (०). Data is represented as (a) Nyquist, (b) Bode modulus, and (c) phase angle from $10^{<\mathrm{M}->1}$ to $10^{5 \wedge \wedge} \mathrm{Hz}$.

Figure $^{\wedge \wedge} 4$ Ratiometric impedance spectrum of the device upon exposure to DI water $(\boldsymbol{\Delta})$, PBS $\mathrm{pH}^{\wedge \wedge} 7.4(\bullet), 0.1(\boldsymbol{\nabla}), 1(\boldsymbol{\bullet})$ and $5(\diamond) \mathrm{mM}$ ammonia in PBS for $10^{<\mathrm{M}->1}$ to $10^{5 \wedge \wedge} \mathrm{Hz}$.

Figure $\wedge^{\wedge} 5$ Device recovery upon being purged with compressed air for $1^{\wedge} \wedge$ min after the exposure of PBS for $15^{\wedge \wedge} \min$. Baseline $(-), 0^{\wedge \wedge} \operatorname{mM}$ PBS $\left(-^{\wedge}-\right)$, air purge $(-. .-)$.

Figure $^{\wedge} 6$ The effect of sample $\mathrm{pH}$ on ratiometric impedance response of the device over time to $<?>1^{\wedge \wedge} \mathrm{mM}$ (delete? Is mentioned later) $<$ ? $>$ ammonia at $10^{3 \wedge \wedge} \mathrm{Hz} .1^{\wedge \wedge} \mathrm{mM} \mathrm{pH} \mathrm{H}^{\wedge \wedge} 7.4(\circ), 0^{\wedge \wedge} \mathrm{mM}$ $\mathrm{pH}^{\wedge \wedge} 11(\boldsymbol{\nabla}), 1^{\wedge \wedge} \mathrm{mM} \mathrm{pH}^{\wedge \wedge} 11(\bullet)$.

Figure $^{\wedge \wedge} 7$ Measurement of ammonia in PBS from 25 to $200^{\wedge \wedge} \mu \mathrm{M}$ (slope $=0.0043$, intercept $=0.9562$, $\left.\mathrm{R}^{2}=0.9868, n=3\right)$ at $10^{3 \wedge \wedge} \mathrm{Hz}$. Inset shows time-based impedimetric response to ammonia.

Figure $^{\wedge \wedge}$ Baseline absolute impedance of polyaniline sensors $(n=15)$ at $10^{3 \wedge \wedge} \mathrm{Hz}$ for $160^{\wedge \wedge} \mathrm{S}$, ranging from to 354 to $466^{\wedge \wedge} \Omega, 6.9^{\wedge} \% \mathrm{RSD}$. 